\title{
Palinopsia as a Rare Presenting Symptom of Occipital Stroke "Case Report”
}

\author{
Muaz Abdellatif Elsayed*, Mohamed Atif Makdum, Sheena Shirmilon Salim, \\ Nuzrath Salmin Abdul Razak \\ University Hospital Sharjah, Sharjah, UAE \\ Email: ^muaz.elsayed@uhs.ae, M.atif@uhs.ae, S.Shirmilon@uhs.ae, N.Salmin@uhs.ae
}

How to cite this paper: Elsayed, M.A., Makdum, M.A., Salim, S.S. and Razak, N.S.A. (2021) Palinopsia as a Rare Presenting Symptom of Occipital Stroke "Case Report". Case Reports in Clinical Medicine, 10, 203-212.

https://doi.org/10.4236/crcm.2021.107026

Received: June 16, 2021

Accepted: July 19, 2021

Published: July 22, 2021

Copyright ( 2021 by author(s) and Scientific Research Publishing Inc. This work is licensed under the Creative Commons Attribution International License (CC BY 4.0).

http://creativecommons.org/licenses/by/4.0/

\begin{abstract}
Palinopsia is the recurrence or persistence of visual images after cessation of the stimulus. Palinopsia has been associated with a wide variety of etiologies and mechanisms such as drug induced, seizures, migraine, psychiatric conditions, head trauma and structural lesions in the brain. We report a case of occipital stroke who presented with oscillating palinopsia. Sudden-onset palinopsia is a very rare symptom of stroke, but it must be recognized early as it is a highly time dependent, and potentially treatable condition. A 57-year-old woman with a history of poorly controlled type 2 diabetes, hyperlipidemia, and hypertension presented with sudden onset right sided palinopsia with images of her face and right forearm with hand, occurring several times in a day, lasting for a few minutes each time, and appearing in the same location each time. There are few case reports in literature secondary to stroke and what makes it interesting also is that she reported persistence of image even when her eyes are closed. Moreover, the sudden onset is not common in literature. The Magnetic Resonance Imaging (MRI) of the brain showed evidence of left Posterior Cerebral Artery (PCA) territorial (Fusiform gyrus) subacute ischemic infarction. Visual field assessment showed a right homonymous hemianopia. She was treated with aspirin and clopidogrel. The frequency and intensity of palinopsia reduced subsequently. The face image disappeared in the first week while the forearm image disintegrated gradually over the next few weeks. Palinopsia may be a rare presenting symptom of stroke. It is important for the clinician or emergency room doctors to be alert to the possibility of undiagnosed structural neurological lesions like occipital stroke and differentiate it from non-organic causes, as prompt management may reduce the risk of visual or motor disability.
\end{abstract}

\section{Keywords}

Palinopsia, Oscillating, Occipital Stroke, Hemianopia, Case Report 


\section{Introduction}

Visual perseveration or palinopsia is a term that describes multiple types of visual symptoms characterized by recurrence or persistence of visual images after cessation of the stimulus. This category of palinopsia represents a dysfunction in visual memory and is caused by posterior cortical lesions or seizures. It can be classified as hallucinatory and illusory palinopsia which can last for various durations. Sudden-onset palinopsia is a very rare symptom of stroke, but must be recognized as stroke, as it is a treatable condition, and the treatment is highly time dependent. This patient is unique in her sudden onset symptoms, vascular aetiology and persistence of image when eyes are closed.

\section{Case Presentation}

A 57-year-old, right-handed lady was referred to the neurology outpatient department (OPD) for evaluation with sudden onset visual symptoms. She had a history of poorly controlled diabetes mellitus, hypertension and dyslipidaemia. There was family history of diabetes mellitus, hypertension and dyslipidaemia. She had a stable psycho-social history. Four days prior to presentation at the neurology OPD, the patient had attended the emergency room for raised blood pressure $(200 / 94 \mathrm{mmHg})$. She had no symptoms at that point and her blood pressure was brought under control with medications. Computed tomography of the brain at that time did not reveal any pathological findings. She complained of blurring of vision the next morning. She underwent an ophthalmological examination which was unremarkable and patient was reassured and sent home.

However, on day 3 , she noted that the blurring of vision had worsened. She further stated that she specifically saw her right hand and forearm on the left upper quadrant of the temporal field while her right upper limb was actually on her knee or over her chest. This occurred several times in a day, lasted for few seconds and the image was static, vivid and had clear contours. The image was visible even when her eyes were closed. Occasionally, she saw her face in the same area of the visual field. This was short lasting and the image was not distorted. She also noted that she was unable to see people standing in her right temporal field. The patient did not report any visual distortions or alterations in colours or micropsia, macropsia or metamorphosia. She also did not report any associated headaches, eye pain, impaired consciousness, or awareness and she was not on any new medications. The patient was very distressed by her symptoms and sought medical attention.

Her ophthalmological assessment showed that the best corrected visual acuity using the Snellen chart was 6/15 in the right eye and 6/30 in the left eye. The intra ocular pressure was normal in both eyes. Colour vision in both eyes was normal. Anterior segment examination revealed that the right pupil was mid-dilated with grade one relative afferent pupillary defect. There was no papilloedema. Both fundi showed moderate non-proliferative diabetic retinopathy with macular edema. The ocular coherence tomography of the macula in both eyes showed 
mild cystoid spaces corresponding to the macular edema with a left vitreomacular traction. She was referred to the neurologist for evaluation of her symptoms.

Neurological examination revealed the presence of an isolated right homonymous hemianopia and absent tendon reflexes. The Magnetic Resonance Imaging (MRI) of the brain (Figure 1) showed an evidence of left Posterior Cerebral Artery (PCA) territorial (Fusiform gyrus) subacute ischemic infarction with evidence of minute haemorrhagic transformation. There was a secondary tonsilar herniation plus the sequelae of chronic lacunar infarcts and involutional brain changes.

Figure 2 shows the findings on the Computed Tomographic Angiography (CTA). There was evident proximal narrowing of the P2 \& P3 segments of the left posterior cerebral artery with total occlusion of its continuation as the P4 segment. There were mild to moderate atherosclerotic changes of the aortic arch with bilateral carotid bulb calcified atheromatous plaques which were more prominent on the left side. The anterior communicating and left posterior corticated artery were hypoplastic. There was a slight narrowing of the M3 segment of the left middle cerebral artery (MCA).
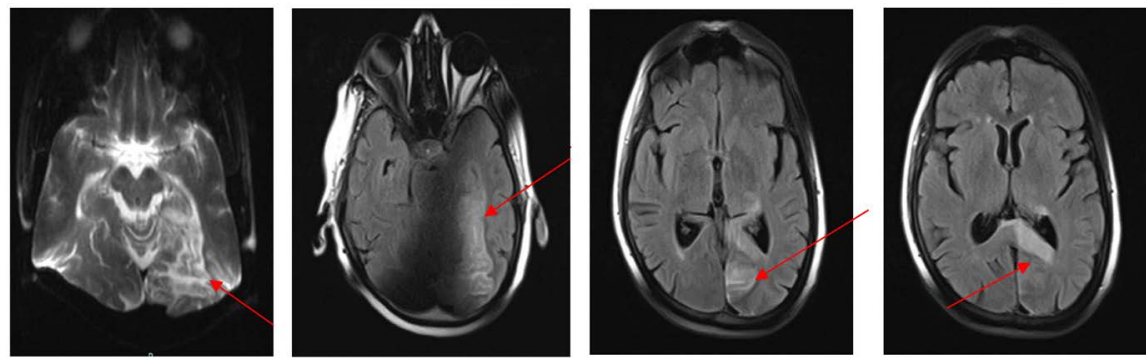

Figure 1. Brain MRI in different sequences showing hyperacute infarction showed by the red arrows on the left occipital lobe.
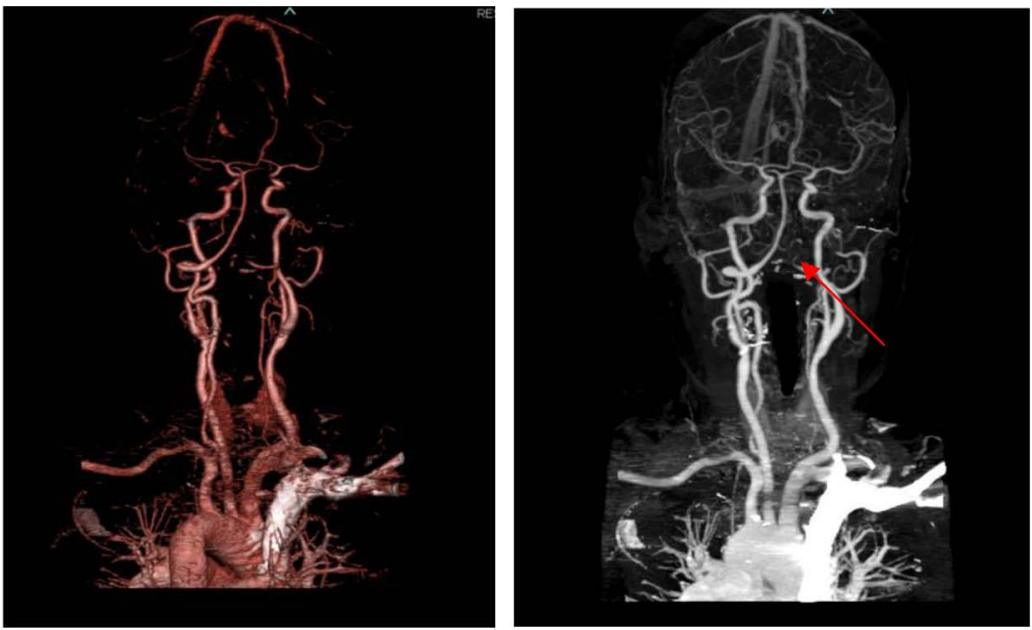

Figure 2. Computed tomography angiography of cerebral vessels showing total occlusion of its continuation as the P4 segment shown as absent artery compared to the right side as shown by red arrows. 
Laboratory investigations including complete blood counts, C-reactive peptide, Vitamin B12, liver, renal, thyroid function tests and coagulation profile were within normal limits. Serum homocysteine levels were marginally high. Lipid profile revealed high triglycerides and low HDL values. Urine analysis revealed microalbuminuria. The antinuclear antibody (ANA) test and the Antineutrophil cytoplasmic antibodies (ANCAs) (both cytoplasmic (c-ANCA) or perinuclear (p-ANCA)) were negative.

Figure 3 demonstrates the electroencephalogram (EEG) record which showed a left posterior occipital and temporal sharp contour wave form discharges, sharp wave discharges and slow waves which were centered at the posterior electrode. The run of discharges disrupted the background alpha rhythm and its field extended to left posterior electrodes in the temporo-occipito-parietal lobes (Low frequency time constant LFT1Hz \& High frequency filter HFF $70 \mathrm{~Hz}$ ). The echocardiogram was unremarkable with no significant arrhythmias or Atrioventricular (AV) block.

Her Visual field assessment showed a right homonymous hemianopia (Figure 4). Visual evoked potentials were within normal limits.

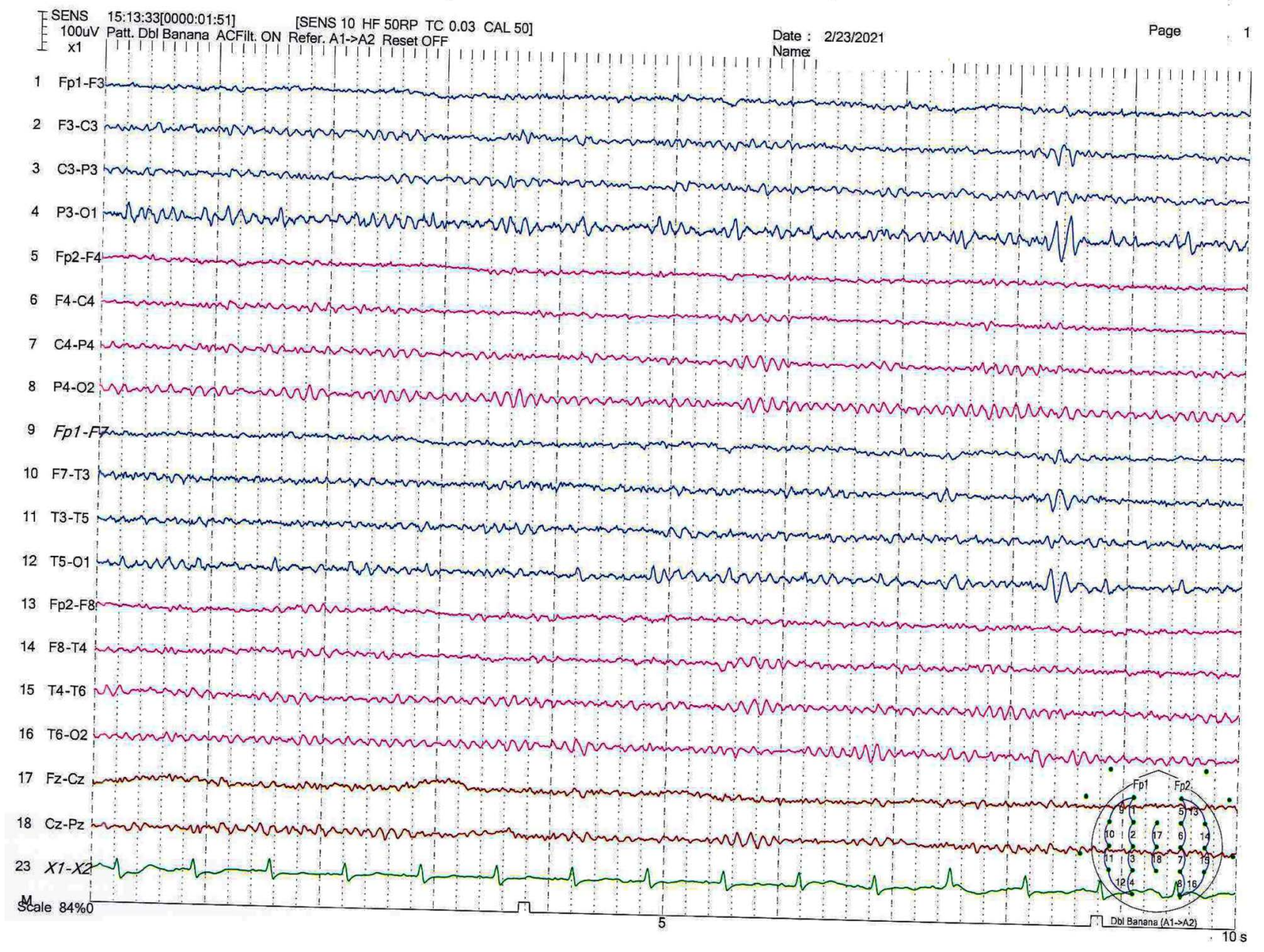

Figure 3. EEG. 

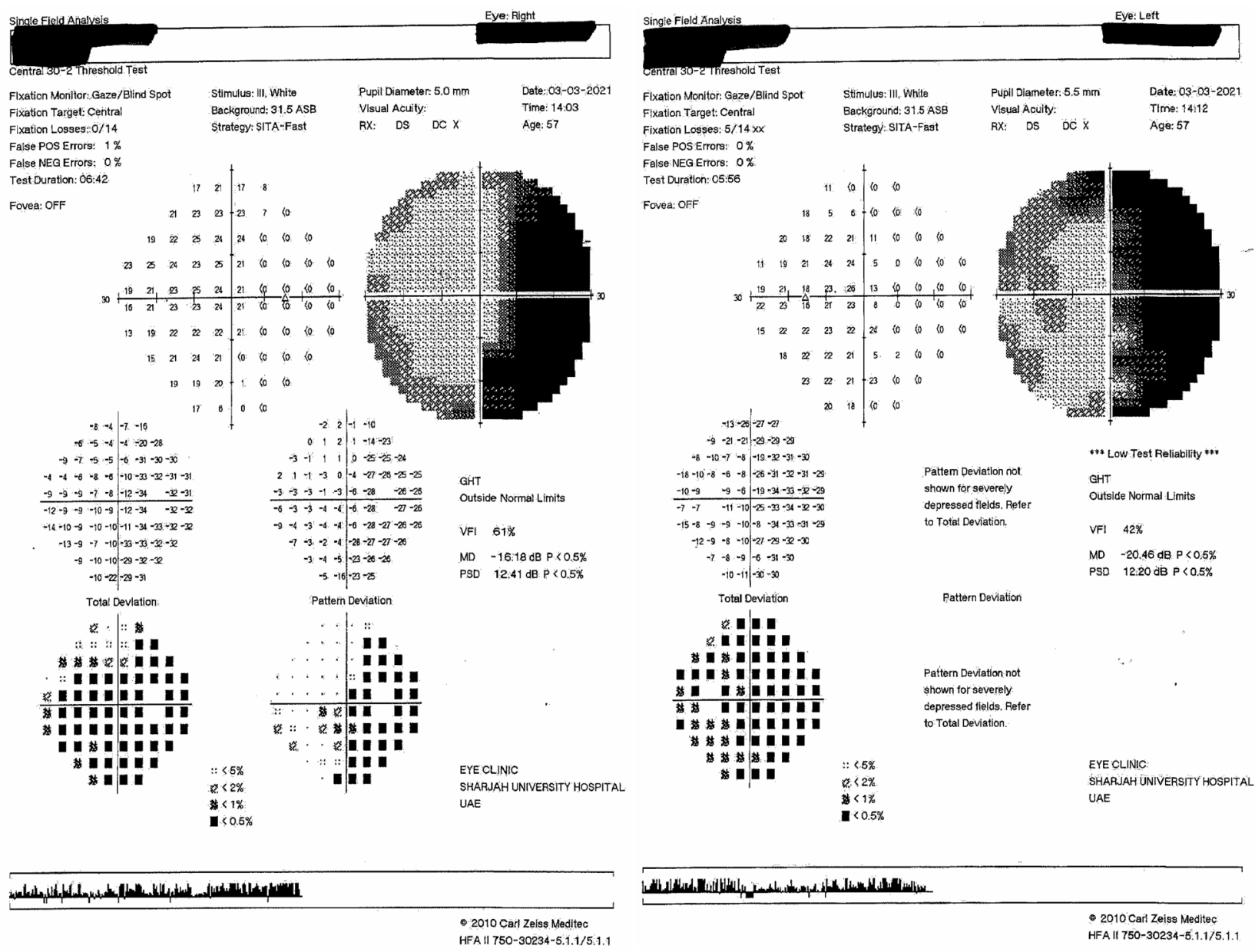

Figure 4. Visual field assessment.

Following completion of this extensive, multi-disciplinary work up, she was diagnosed as a case of palinopsia due to occipital ischemic stroke secondary to left posterior cerebral artery (P4) thrombotic occlusion. Her medical back ground was the main risk factor.

The patient was started on high dose aspirin $(300 \mathrm{mg})$ daily for one week along with statins, rehydration, Omega 3, Citicholine and NeuroAid capsules (proprietary blend of 9 herbal ingredients). The patient remained on regular follow up with the neurology and ophthalmology OPD. One week later, she reported that the afterimages of her hand and forearm were less frequent and less intense. At times, the image also appeared to disintegrate. The afterimages of her face disappeared after a week. Her hemianopia, however did not improve. She was reassured and started on Clopidogrel $75 \mathrm{mg}$ and low dose aspirin $100 \mathrm{mg}$ along with her other medications. Clopidogrel was discontinued after a few days as she developed sores/ulcers in the mouth. The patient was followed up for 4 months in the neurology/Ophthalmology OPDs. Her palinopsia did not relapse, had no hemiplegia, but her hemianopia improved mildly depending on the objective visual field assessment. The diabetic, lipid and hypertensive levels were well controlled. 


\section{Discussion}

We presented a patient with long standing risk factors for ischaemic stroke having repeated episodes of palinopsia as a major presenting symptom of stroke. Palinopsia is always indicative of some systemic dysfunction. It is important for the clinician to be alert to the possibility of undiagnosed neurologic disease, as it could be life-saving.

This case is unique in that palinopsia was a presenting feature of stroke. The well-coordinated assessments lead to critical diagnoses that met the patient's concerns. Our patient was clearly satisfied with the management of palinopsia and we outlined stroke prognosis depending on the risk factors and size of infarction on the MRI brain. This affected her visual field capacity. When palinopsia is due to structural disease, palinopsia usually occurs in patients with impaired vision who are not entirely -blind and usually tends to occur transiently during the progression or resolution of visual field defect [1]. Sun Yuan-Ting et al reported a case with a sequential appearance and disappearance within two days of homonymous hemianopsia, palinopsia and metamorphopsia in the defective visual field, nine months after the stroke [2]. They speculated that sequential hemodynamic changes in the right occipital area, from hypoperfusion to transient hyperperfusion and finally to normal perfusion could have precipitated these symptoms. Weber et al described a case of a 77-year-old man with ischaemic risk factors who presented with isolated intermittent palinopsia lasting for a few hours [3]. There were no other focal neurological or associated symptoms and his neurological examination was unremarkable. He was managed as a case of acute occipital lobe stroke.

The palinopsia in our patient was not precipitated by the presence of an obvious visual stimulus and was not associated with migraine. Migraine is the most common single cause of visual hallucinations and illusions, and palinopsia can be present either before or after a migraine attack. Kalita et al. reported that around $10 \%$ of migraineurs had palinopsia and they tend to be more frequent and persistent than in normal individuals [4]. The increased frequency of palinopsia in migraineurs may suggest an enhanced physiological function of cortical neurons in the migraine. Takayama et al reported a case of a 55-year-old male patient with long-standing migraine history who developed an abscess in the right occipital lobe [5]. The patient developed episodes of palinopsia accompanied with migraine attacks after healing of the occipital lesion. The authors hypothesized that migraine potentiates the effect of cortical lesions in generation of palinopsia and palinopsia occurred only when the neocortical excitability exceeded a certain limit by severe migraine attack.

Palinopsia can occur as manifestation of epilepsy and seizures of the visual processing and memory centres of the brain might be the underlying cause of palinopsia [6]. Palinopsia may occur during a seizure aura, the ictal phase, and postictally and anticonvulsive therapy has also been found to reduce palinopsia in symptomatic patients [7]. Occasionally palinopsia may be the only symptom 
of the seizure, without any motor or sensory symptoms [7]. Our patient did not have any clinical symptoms of epilepsy and her EEG changes may be related to the structural damage over the occipital cortex. However, the cortical irritation or hyperexcitability due to stroke may also have provoked the epileptic phenomenon and the two cannot be easily distinguished. However, the isolated palinopisa, the duration of the event and relapses during the day without other features of epilepsy and reduction of symptoms without anti-convulsant therapy makes it less likely to be a manifestation of epilepsy. Systemic disease can result in epilepsy, as reported by Kondziella and Maetzel, wherein a patient experienced palinopsia associated with epileptic seizures caused by an occipital lobe glioblastoma [8].

Episodes of palinopsia may also occur due to disturbances of peripheral vestibular function. Stafuzza et al reported a patient with episodes of palinopsia after the functional loss of the 3 semicircular canals of the right ear with preservation of the otolithic function [9]. The abnormal temporal integration of the visual and vestibular inputs may result in palinopsia if there is a delay in the vestibular inputs [9]. The absence of vestibular symptoms and normal hearing function in our patient makes it unlikely to be a causal factor.

Palinopsia has generally been described in reference to static afterimages. In our case too, the patient reported static images of her forearm and hand. Lahiri et al reported a case of kinetic palinopsia with images of a car moving backwards in his left visual field [10]. Similar to our case, their patient also had an associated field defect (left homonymous hemianopia) due to right occipital arteriovenous malformation (AVM) affecting the lingual gyrus and right occipital lobe. Palinopsia commonly occurs in the setting of posterior visual pathway deafferentation causing homonymous visual field deficits and it is most often hallucinatory palinopsia. This palinoptic mechanism is thought to be similar to visual release hallucinations (Charles-Bonnet syndrome), which are due to neuronal hyperexcitabiity, often from ocular vision loss [7]. Whereas hallucinatory palinopsia occurs due to focal cortical hyperexcitability from cortical visual loss. Visual release hallucinations can be distinguished from palinopsia by the content of the images (whether the formed image or scene actually occurred).

Palinopsia has been reported in Creutzfeldt-Jakob disease (CJD), Leber's Hereditary optic neuropathy, optic neuritis, perilesional hyperperfusion, multiple sclerosis, ceroid lipofuscinosis, Glycine receptor antibody syndrome, nonketotic hyperglycemia, tumors and leukemias [6] [7] [10] [11]. There were no associated neurodegenerative processes or other systemic diseases which may be a likely etiology for the palinopsia in our patient. There was no cognitive decline or ataxia as seen in palinopsia in ceroid lipofuscinosis [11]. Palinopsia usually results from a lesion in the dominant hemisphere in $25 \%$ of cases [9], as seen in our patient. Authors have reported a case of a glioblastoma tumor in the non-dominant occipito-temporal region, wherein the pattern of palinopsias was a complex scene of the family members and room contents [12]. In contrast, our patient had static palinopsia with afterimages of his own forearm and hand. 
Palinopsia has been found to be brought on by a variety of prescribed medications (Topiramate, clomiphene citrate, Zosuquidar, Nefazodone, Interleukin 2 therapy, trazodone) and illicit drugs (marijuana, mescaline, lysergic acid dyethilamide, 3,4-methylenedioxymethamphetamine [6] [7] [13] [14] [15] and most are illusory palinopsia. These symptoms typically occur during drug initiation or dose increase and resolve after drug discontinuation. Clomiphen citrate an infertility drug can result in palinopsia that may persist for years after use of the drug [13] [14]. The illusory palinopsia induced by drugs is mainly caused by its action on the serotonergic system and serotonin depletion leads to cortical hyperexcitability. In our case, a thorough history taking and inventory of medications, both past and present was done to rule out any influence of drug induced palinopsia. Also, the treatment for ischaemic stroke reduced the frequency and intensity of palinopsia. The face image disappeared in the first week while the arm image disintegrated gradually over the next few weeks.

Palinopsia in our patient was also not associated with visual snow which is characterized by the perception of TV static-like "snow" in the entire visual field [16]. The absence of vestibular symptoms and normal hearing function of this patient makes it unlikely to be associated with vestibular dysfunction which may provoke palinopsia in few cases [9]. Selective intra cerebral stimulation of the right lateral fusiform gyrus may result in partial or superimposed palinopsia on other images (facial palinopsia) [17]. Involvement of fusiform gyrus in stroke may result in facial palinopsia, i.e. an individual face percept superimposed on the non-face stimulus. This might explain the face image observed by our patient.

\section{Conclusion}

Palinopsia may be a presenting symptom of stroke. It is important for the clinician or Emergency room doctors to be alert to the possibility of undiagnosed structural neurological lesions like occipital stroke and differentiate it from nonorganic causes, as prompt management may reduce the risk of visual or motor disability. Palinopsia is an unusual symptom with which many clinicians outside neurology may be unfamiliar and often requires interaction from multiple disciplines including neurology, eye care, and psychiatry. Those experiencing palinopsia often may be distressed and reluctant to describe their rather disturbing symptoms clearly to the attending physician. It is imperative for doctors to be aware of this condition, recognize associated factors and elicit the information by asking correct questions to the patient. In addition, it is important to address their concerns and reassure them that what they experience is a well-documented phenomenon and may be more common than it is perceived to be.

\section{Ethical Approval and Consent to Participate}

This work follows the guidelines for human studies and was conducted ethically in accordance with the World Medical Association Declaration of Helsinki. 
Written informed consent was obtained from the patient for reporting and publication of this case report and any accompanying images and the relevant approval was obtained from the ethical committee of the University Hospital Sharjah.

\section{Consent for Publication}

The consent for publication was obtained from both the patient and the University Hospital Sharjah ethical committee.

\section{Availability of Supporting Data}

The date is stored in the electronic database of the University Hospital Sharjah under the confidentiality act.

\section{Authors Contributions}

Dr. M.E was responsible for the literature review, neurological assessment of the patient, writing of discussion and references. Dr. M.A had evaluated the patient and did the ophthalmological work up personally. He contributed to manuscript writing in his area of expertise. Mrs. S.S and Mrs. N.S shared the effort of electrophysiology studies while all authors edited the English language of the manuscript and have approved the final draft of the manuscript before sending for publication.

\section{Acknowledgements}

We thank inputs from our colleagues to help editing this paper specially Dr. Mohamed Ayas the audiologist.

\section{Conflicts of Interest}

The authors have no competing interest to declare.

\section{References}

[1] Bender, M.B., Feldman, M. and Sobin, A.J. (1968) Palinopsia. Brain: A Journal of Neurology, 91, 321-338. https://doi.org/10.1093/brain/91.2.321

[2] Sun, Y.T. and Lin, C.C. (2004) Sequential Appearance and Disappearance of Hemianopia, Palinopsia and Metamorphopsia: A Case Report and Literature Review. Acta Neurologica Taiwanica, 13, 77-83.

[3] Kataoka, H. and Ueno, S. (2009) Cerebral Polyopia and Palinopsia in a Patient with Occipital Lobe Epilepsy. Epilepsy \& Behavior, 14, 684-686. https://doi.org/10.1016/j.yebeh.2009.01.026

[4] Kalita, J., Uniyal, R. and Bhoi, S.K. (2016) Is Palinopsia in Migraineurs an Enhanced Physiological Phenomenon? Cephalalgia: An International Journal of Headache, 36, 1248-1256. https://doi.org/10.1177/0333102415625610

[5] Takayama, Y., Iwata, J. and Kojima, A. (2019) Palinopsia Accompanied with Migraine Attack after Having Occipital Brain Abscess. Neuro-Ophthalmology, 43, 327-329. https://doi.org/10.1080/01658107.2018.1519581 
[6] Abert, B. and Ilsen, P.F. (2010) Palinopsia. Optometry, 81, 394-404. https://doi.org/10.1016/j.optm.2009.12.010

[7] Gersztenkorn, D. and Lee, A.G. (2015) Palinopsia Revamped: A Systematic Review of the Literature. Survey of Ophthalmology, 60, 1-35. https://doi.org/10.1016/j.survophthal.2014.06.003

[8] Kondziella, D. and Maetzel, H. (2006) The Sting in the Tail: Syncope and Palinopsia. Journal of Neurology, 253, 657-658. https://doi.org/10.1007/s00415-006-0012-7

[9] Stafuzza, C., Landis, T. and Guyot, J.P. (2018) Palinopsia Following Acute Unilateral Partial Vestibular Deafferentation: A Case Report. Frontiers in Neurology, 9, 773. https://doi.org/10.3389/fneur.2018.00773

[10] Lahiri, D. (2020) Palinopsia. Cortex. A Journal Devoted to the Study of the Nervous System and Behavior, 133, 399. https://doi.org/10.1016/j.cortex.2020.10.004

[11] Kamate, M., Detroja, M. and Hattiholi, V. (2019) Neuronal Ceroid Lipofuscinosis Type-11 in an Adolescent. Brain \& Development, 41, 542-545. https://doi.org/10.1016/j.braindev.2019.03.004

[12] Huang, M., Baskin, D.S. and Fung, S. (2016) Glioblastoma Presenting with Pure Alexia and Palinopsia Involving the Left Inferior Occipital Gyrus and Visual Word form Area Evaluated with Functional Magnetic Resonance Imaging and Diffusion Tensor Imaging Tractography. World Neurosurgery, 89, 725.e5-725.e10. https://doi.org/10.1016/j.wneu.2015.12.071

[13] Venkatesh, R., Gujral, G.S., Gurav, P., Tibrewal, S. and Mathur, U. (2017) Clomiphene Citrate-Induced Visual Hallucinations: A Case Report. Journal of Medical Case Reports, 11, 60. https://doi.org/10.1186/s13256-017-1228-0

[14] Choi, S.Y., Jeong, S.H. and Kim, J.S. (2017) Clomiphene Citrate Associated with Palinopsia. Journal of Neuro-Ophthalmology: The Official Journal of the North American Neuro-Ophthalmology Society, 37, 220-221.

https://doi.org/10.1097/WNO.0000000000000532

[15] Yun, S.H., Lavin, P.J., Schatz, M.P. and Lesser, R.L. (2015) Topiramate-Induced Palinopsia: A Case Series and Review of the Literature. Journal of Neuro-Ophthalmology: The Official Journal of the North American Neuro-Ophthalmology Society, 35, 148-151. https://doi.org/10.1097/WNO.0000000000000216

[16] Berkowitz, E., River, Y., Digre, K., Tiosano, B. and Kesler, A. (2020) Visual Snow: A Case Series from Israel. Case Reports in Ophthalmology, 11, 205-211. https://doi.org/10.1159/000508602

[17] Jonas, J., Brissart, H., Hossu, G., Colnat-Coulbois, S., Vignal, J.-P., Rossion, B. and Maillard, L. (2018) A Face Identity Hallucination (Palinopsia) Generated by Intra Cerebral Stimulation of the Face-Selective Right Lateral Fusiform Cortex. Cortex: $A$ Journal Devoted to the Study of the Nervous System and Behaviour, 99, 296-310. https://doi.org/10.1016/j.cortex.2017.11.022 